\title{
An Interactive Antimicrobial Activity of Embelica officinalis Gaertn. Fruit Extracts and Conventional Antibiotics against Some Bacterial Triggers of Autoimmune Inflammatory Diseases
}

\author{
Adrian Hutchings ${ }^{1}$, Ian Edwin Cock $^{1,2^{*}}$
}

\section{Adrian Hutchings ${ }^{1}$, Ian Edwin Cock ${ }^{1,2^{*}}$}

\section{'School of Natural Sciences, Nathan Campus, Griffith University, 170 Kessels Rd, Nathan, Queensland, AUSTRALIA. 1,2Environmental Futures Research Institute, Nathan Campus, Griffith University, 170 Kessels Rd, Nathan, Queensland, AUSTRALIA. \\ Correspondence}

Dr lan Edwin Cock

Environmental Futures Research Institute, Nathan Campus, Griffith University, 170 Kessels Rd, Nathan, Queensland, AUSTRALIA.

Phone no : +61737357637

E-mail: i.Cock@griffith.edu.au

History

- Submission Date: 26-02-2018;

- Revised Date: 14-03-2018;

- Accepted Date: 03-05-2018

DOI : 10.5530/pj.2018.4.108

Article Available online http://www.phcogj.com/v10/i4

\section{Copyright}

(C) 2018 Phcog.Net. This is an openaccess article distributed under the terms of the Creative Commons Attribution 4.0 International license.

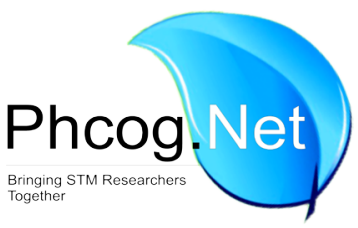

\begin{abstract}
Background: Embelica officinalis Gaertn. is an Indian plant which is known for its therapeutic properties. It is especially well known as a component of the Ayuverdic medicine Triphala. This study focuses on the growth inhibitory activity of $E$. officinalis fruit extracts against some bacterial triggers of autoimmune inflammatory diseases, both alone and in combination with conventional antibiotics. Methods: E. officinalis fruit powder was extracted with solvents of varying polarity and screened for bacterial growth inhibition by disc diffusion assay. The minimum inhibitory concentration (MIC) was quantified by both liquid dilution and disc diffusion techniques. To screen for combinatorial effects, the E. officinalis fruit extracts were combined with a range of conventional antibiotics and tested against each bacteria using a liquid dilution assay. Toxicity was examined using Artemia nauplii and HDF bioassays. Results: The ethyl acetate E. officinalis fruit extract displayed the strongest growth inhibitory activity against all of the bacterial triggers of autoimmune inflammatory disease. This extract was a particularly potent inhibitor of $P$, aeruginosa growth, with an MIC values as low as $264 \mu \mathrm{g} / \mathrm{mL}$. The ethyl acetate extract was also a moderate to strong growth inhibitor of $P$. mirabilis, $K$. pneumonia and $A$. baylyi, with MIC values generally $1000-1500 \mu \mathrm{g} / \mathrm{mL}$. The methanolic and aqueous extracts also inhibited the growth of all bacteria, although generally with only moderate to low activity. Whilst no synergistic interactions were detected in combinations containing the $E$. officinalis fruit extracts and conventional antibiotics, a number of combinations produced additive effects. These combinations are beneficial as they provide enhanced antibacterial efficacy compared to treatment with the antibiotic or extract components alone. No antagonistic interactions were detected. Therefore, use of the extracts in combination with conventional antibiotics would not compromise the antibiotics efficacy. All extracts were nontoxic in the Artemia nauplii and HDF toxicity assays, further indicating their potential for medicinal use. Conclusion: The $E$. officinalis fruit extracts were moderate inhibitors of the bacterial triggers of selected autoimmune inflammatory diseases. Furthermore, the extracts potentiated the activity of chloramphenicol and tetracycline against otherwise resistant bacterial strains. Isolation of the active compounds and the potentiating agents may be beneficial in antibiotic drug design.

Key words: Amla, Indian gooseberry, Synergy, Multi-drug resistant bacteria, Combinational
\end{abstract} therapies, Rheumatoid arthritis, Ankylosing spondylitis, Multiple sclerosis.

\section{INTRODUCTION}

The incorrect and over use of antibiotics has resulted in a dramatic rise in the prevalence of antibiotic resistant bacterial pathogens and several extremely (XDR) or totally drug resistant (TDR) pathogens have recently been reported. ${ }^{1}$ There are now limited therapeutic options for the diseases caused by these pathogens and the development of alternative antibiotic chemotherapies is considered by the World Health Organisation (WHO) to be one of the most serious challenges facing medical science. ${ }^{2}$ Previously, much antibiotic drug development has focussed on microbial derived compounds with antibiotic activity (e.g. penicillin). Whilst this approach has been effective in the discovery and development of new antibiotics, it is unlikely to yield the same wealth of new drugs into the future. The majority of common bacteria and fungi have already been screened, and where suitable, promising compounds have undergone clinical trials. A relatively small proportion of these compounds were deemed suitable for further development and commercialisation as antibiotic drugs. ${ }^{3}$ The frequency of discovery for new bacterial and fungal species continues to decrease. Furthermore, many of the newly discovered microbes grow in extreme and/or unusual environments with lower competition and are therefore unlikely to be as abundant in antibiotic compounds. Thus, whilst screening newly discovered bacterial and fungal species for novel antibiotic compounds
Cite this article: Hutchings A, Cock IE. The Interactive Antimicrobial Activity of Embelica officinalis Gaertn. Fruit Extracts and Conventional Antibiotics against some Bacterial Triggers of Autoimmune Inflammatory Diseases. Pharmacog J. 2018;10(4):654-62. 
should not be ignored, future antibiotic discovery will increasingly be reliant on developing new discovery sources and/or treatment modalities. Alternatively, synthetic drug design relies on adapting known chemical scaffolds by the modification and the alteration of functional groups (e.g. the synthesis of methicillin from penicillin). ${ }^{4}$ This has also proved to be a fruitful source of new antibiotics with greater activity and/ or bioavailability. ${ }^{4}$ However, new scaffolds are required to allow synthetic chemists to continue to adapt and develop new antibiotics.

Traditional medicines have great potential for antimicrobial drug development. Indeed, thousands of studies have been published in recent years describing the antibacterial properties of medicinal plant extracts and natural products isolated from plants. Despite this, relatively few plant derived antibiotic compounds are widely used clinically. Many pure plant compounds have relatively low activity alone and require synergistic interactions with other components to potentiate their antibacterial activity. ${ }^{5}$ A combinational approach that allows synergistic interaction between plant extracts (or pure plant compounds) and conventional antibiotics may therefore be more effective in combatting bacterial pathogens, especially against antibiotic resistant strains. ${ }^{6,7}$ A combination of plant extracts/isolated compounds with conventional antibiotics may also prove to be an effective way to rapidly develop new antibiotic chemotherapies. ${ }^{5}$ Developing new drugs requires years of extensive and costly testing. However, combinational therapy can potentially restore an existing drug to a state of significantly reduced resistance, thereby bypassing this lengthy and expensive process. ${ }^{5}$ Further advantages of synergistic interactions may include increased efficiency, reduced side effects, increased stability and bioavailability and the need for lower doses in comparison to synthetic alternatives. ${ }^{5}$

The Indian medicinal system Ayurveda uses a variety of natural plant and fruit products to treat numerous ailments. ${ }^{8-10}$ Triphala is an Ayuverdic formulation used to treat a variety of illnesses, including uses as a immunostimulant, as well as for diabetes and gastrointestinal problems. ${ }^{9}$ It also has anti-inflammatory and antibacterial properties and thus has potential against autoimmune inflammatory diseases. Triphala is derived from the dried fruit from three plant species: Terminalia bellerica (Gaertn.) Roxb, Terminalia chebula Retz., and Embilica officinalis Gaertn. Triphala, and each of those plants, are widely used as an anti-inflammatory, antioxidant and antimicrobial agents. ${ }^{10,11}$ Each of the individual plant components have been shown to have therapeutic effects on respiratory and gastrointestinal problems when used alone, and it has even been reported that the active compounds found in Triphala are effective in treating some cancers. ${ }^{9,10}$

E. officinalis (Synonyms Phyllanthus emblica L. Cicca emblica (L.) Kurz, Diasperus emblica (L.) Kuntze, Dichelactina nodiculis Hance; commonly known as Amla or Indian gooseberry) is widely used in Indian Ayuverda and Unani traditional medicine systems to treat a variety of diseases. ${ }^{12}$ It is particularly useful for its immunomodulatory, anti-inflammatory, analgesic and antimicrobial properties and therefore has potential for the prevention and treatment of chronic inflammatory diseases. It is also beneficial in the treatment of cancer and diabetes, as well as gastrointestinal, hepatic, cardiovascular diseases. The fruits of E. officinalis contain tannins, polyphenolics and alkaloids. ${ }^{13,14}$ In particular, the tannins gallic acid, 3-ethylgallic acid, ellagic acid, chebulinic acid, chebulagic acid and corilagin and have been reported to be present in significant concentrations in E. officinalis fruit. ${ }^{14}$ Similarly, E. officinalis fruit also contain appreciable levels of the flavonoids quercetin, kaempferol and apigenin. ${ }^{13}$ These phytochemicals contribute to the high antioxidant capacity and free radical scavenging activity, anti-cancer activity, antibacterial, antifungal, antiviral and anti-inflammatory activities, as well as the wound healing and immunomodulatory activities associated with E. officinalis fruit. Furthermore, E. officinalis extracts have been reported to have potent antibacterial activity against a panel of pathogenic bacteria. ${ }^{15}$ That study reported that aqueous E. officinalis fruit extracts were particularly potent inhibitors of several Staphylococcus spp., Micrococcus spp. and Bacillus spp. However, the extracts were screened at a single, relatively high concentration and MIC values were not determined, making comparison of the efficacy of these extracts with other studies impossible. Despite the earlier antibacterial studies, E. officinalis fruit extracts are yet to be screened for the ability to inhibit the growth of the bacterial triggers of autoimmune inflammatory diseases. Four bacterial strains were selected for screening against the E. officinalis fruit extracts based on their ability to trigger autoimmune diseases: Proteus mirabilis is a trigger for rheumatoid arthritis; Klebsiella pnuemoniae is a bacterial trigger for ankylosing spondylitis; and Acinetobacter baylyi and Pseudomonas aeruginosa can trigger multiple sclerosis in genetically susceptible people. ${ }^{16,17}$ Furthermore, E. officinalis extracts are yet to be tested for potentiating activity in combinational studies with conventional antibiotics. This study aimed to investigate the growth inhibitory activity of E. officinalis fruit extracts against P. mirabilis, K. pneumonia, A. baylyi and P. aeruginosa, alone and in combination with conventional antibiotics to evaluate their interactive effects.

\section{MATERIALS AND METHODS}

\section{Plant material and extraction}

The Embelica officinalis Gaertn. fruit used in this study were sourced from verified trees in India by Noodles Emporium, Australia and supplied as a dried and ground powder. A voucher sample (EOF2016clah) has been stored at the School of Natural Sciences, Griffith University, Australia. Individual $1 \mathrm{~g}$ quantities of the material were weighed into separate tubes and $50 \mathrm{~mL}$ of methanol, deionised water, chloroform, hexane or ethyl acetate were added. All solvents were obtained from Ajax and were AR grade. The ground plant materials were individually extracted in each solvent for $24 \mathrm{hr}$ at $4^{\circ} \mathrm{C}$ with gentle shaking. The extracts were then filtered through filter paper (Whatman No. 54) under vacuum, followed by drying by rotary evaporation in an Eppendorf concentrator 5301 . The resultant extracts were weighed and redissolved in $10 \mathrm{~mL}$ deionised water (containing $1 \%$ DMSO).

\section{Qualitative phytochemical studies}

Phytochemical analysis of the E. officinalis fruit extracts for the presence of saponins, phenolic compounds, flavonoids, phytosterols, triterpenoids, cardiac glycosides, anthraquinones, tannins and alkaloids was conducted by previously described assays. ${ }^{18-20}$

\section{Antibacterial screening}

\section{Conventional Antibiotics}

Penicillin-G (potency of $1440-1680 \mu \mathrm{g} / \mathrm{mg}$ ), chloramphenicol ( $\geq 98 \%$ purity by HPLC, erythromycin (potency $\geq 850 \mu \mathrm{g} / \mathrm{mg}$ ), gentamicin (potency of $600 \mu \mathrm{g} / \mathrm{mg}$ ), and tetracycline ( $\geq 95 \%$ purity by HPLC) were purchased from Sigma-Aldrich, Australia and were used as controls for the microplate liquid dilution assay. All antibiotics were prepared in sterile deionised water at stock concentrations of $0.01 \mathrm{mg} / \mathrm{mL}$ and stored at $4^{\circ} \mathrm{C}$ until use. For the disc diffusion studies, penicillin $(20 \mu \mathrm{g})$, nystatin (100 Units), ciprofloxacin $(2.5 \mu \mathrm{g})$ and chloramphenicol $(10 \mu \mathrm{g})$ standard discs were obtained from Oxoid Ltd., Australia and used as positive controls.

\section{Bacterial cultures}

All bacterial strains were selected based on their ability to trigger autoimmune inflammatory diseases in genetically susceptible individuals. ${ }^{16,17}$ Reference strains of Proteus mirabilis (ATCC21721), Klebsiella pneumoniae (ATCC31488), Acinetobacter baylyi (ATCC33304) and Pseudomonas 
aeruginosa (ATCC39324) were purchased from American Type Culture Collection, USA. All bacteria were cultured in nutrient broth (Oxoid Ltd., Australia). Streak nutrient agar (Oxoid Ltd., Australia) plates were tested in parallel to ensure the purity of all bacterial cultures and for sub-culturing. All bacterial cultures were incubated at $37^{\circ} \mathrm{C}$ for $24 \mathrm{~h}$ and were subcultured and maintained in nutrient broth at $4^{\circ} \mathrm{C}$ until use.

\section{Evaluation of antibacterial activity}

The E. officinalis fruit extracts were assessed for antibacterial activity using a modified disc diffusion assay. ${ }^{21,22}$ Penicillin $(20 \mu \mathrm{g})$, nystatin (100 Units), ciprofloxacin $(2.5 \mu \mathrm{g})$ and chloramphenicol discs $(10 \mu \mathrm{g})$ were obtained from Oxoid Ltd., Australia and used as positive controls. Filter discs infused with $10 \mu \mathrm{L}$ of distilled water were used as a negative control.

\section{Minimum inhibitory concentration (MIC) determination}

The minimum inhibitory concentration for each extract was determined using a liquid dilution MIC assay ${ }^{23,24}$ and a solid phase agar disc diffusion MIC assay. ${ }^{25,26}$

\section{Microplate liquid dilution MIC assay}

The MICs of the extracts were evaluated by standard methods. ${ }^{23,24}$ All plates were incubated at $37^{\circ} \mathrm{C}$ for $24 \mathrm{~h}$. P-Iodonitrotetrazolium violet (INT) was obtained from Sigma-Aldrich, Australia and dissolved in sterile deionised water to prepare a $0.2 \mathrm{mg} / \mathrm{mL}$ INT solution. A $40 \mu \mathrm{L}$ volume of this solution was added into all wells and the plates were incubated for a further $6 \mathrm{hr}$ at $30^{\circ} \mathrm{C}$. Following incubation, the MIC was visually determined as the lowest dose at which colour development was inhibited.

\section{Disc diffusion MIC assay}

The minimum inhibitory concentrations (MIC) of the extracts was also evaluated by disc diffusion assay as previously described. ${ }^{25,26}$

\section{Embelica officinalis fruit extract-conventional antibiotic synergy studies \\ Fractional inhibitory concentration (FIC) assessment}

Interactions between the E. officinalis fruit extracts and the conventional antibiotics were examined by determination of the sum of fractional inhibitory concentrations ( $\Sigma$ FIC) for each combination..$^{23,24}$ The FIC values for each component ( $\mathrm{a}$ and $\mathrm{b}$ ) were calculated using the following equations where a represents the plant extract sample and $\mathrm{b}$ represents the conventional antibiotic:

$$
\begin{aligned}
& \mathrm{FIC}(\mathrm{a})=\left(\frac{\mathrm{MIC}[\mathrm{a} \text { in combination with } \mathrm{b}]}{\mathrm{MIC}[\mathrm{a} \text { independently }]}\right) \\
& \mathrm{FIC}(\mathrm{b})=\left(\frac{\mathrm{MIC}[\mathrm{b} \text { in combination with } \mathrm{a}]}{\mathrm{MIC}[\mathrm{b} \text { independently }]}\right)
\end{aligned}
$$

The $\Sigma$ FIC was then calculated using the formula $\Sigma$ FIC $=$ FIC (a) + FIC(b). The interactions were classified as synergistic $(\Sigma \mathrm{FIC} \leq 0.5)$, additive $(\Sigma \mathrm{FIC}$ $>0.5-1.0)$, indifferent $(\Sigma \mathrm{FIC}>1.0-4.0)$ or antagonistic $\left(\sum \mathrm{FIC}>4.0\right){ }^{23,24}$

\section{Toxicity screening}

Two assays were used to assess the toxicity of the individual samples. The Artemia nauplii lethality assay (ALA) was utilised for rapid preliminary toxicity screening, whereas the MTS cellular viability assay was used as a cellular evaluation of toxicity.

\section{Artemia franciscana Kellogg nauplii toxicity screening}

Potassium dichromate $\left(\mathrm{K}_{2} \mathrm{Cr}_{2} \mathrm{O}_{7}\right)$ (AR grade, Chem-Supply, Australia) was prepared in deionised water $(4 \mathrm{mg} / \mathrm{mL})$ and serially diluted in artificial seawater for use as a reference toxin. Toxicity of the E. officinalis fruit extracts, the reference toxin and the conventional antibiotics was assessed using a modified Artemia franciscana nauplii lethality assay. ${ }^{27-29}$ The $\mathrm{LC}_{50}$ with $95 \%$ confidence limits for each treatment was calculated using probit analysis.

\section{Cellular viability assay}

The E. officinalis fruit extracts and conventional antibiotics were screened individually towards normal human primary dermal fibroblasts (HDF) as previously described. ${ }^{30} \mathrm{HDF}$ cells were obtained from American Type Culture Collection (ATCC PCS-201-012). The cells were cultured and maintained in Dulbecco's modified eagle medium (DMEM; Thermo Fisher Scientific, Australia), supplemented with $10 \%$ foetal calf serum (Life Technologies), $50 \mu \mathrm{g} / \mathrm{mL}$ streptomycin (Sigma-Aldrich, Australia) and $50 \mathrm{IU} / \mathrm{mL}$ penicillin (Sigm-Aldricha, Australia). Suspensions of cells which had obtained $80 \%$ confluency were resuspended in fresh media (lacking streptomycin and penicillin supplementation) and $70 \mu \mathrm{L}$ aliquots (containing approximately 5000 cells) were added to individual wells of a 96 well plate. A volume of $30 \mu \mathrm{L}$ of the test extracts or cell media (for the negative control) was subsequently added to individual wells and the plates were incubated at $37^{\circ} \mathrm{C}, 5 \% \mathrm{CO}_{2}$ for $24 \mathrm{hr}$ in a humidified atmosphere. All extracts were screened at $200 \mu \mathrm{g} / \mathrm{mL}$. The cells were then washed in PBS ( $\mathrm{pH}$ 7.2) to remove interference due to sample colour. A volume of $20 \mu \mathrm{L}$ of Cell Titre 96 Aqueous One solution (Promega) was subsequently added to each well and the plates were incubated for a further $3 \mathrm{hr}$. Absorbances were recorded at a test wavelength of $540 \mathrm{~nm}$ and a blank wavelength of $690 \mathrm{~nm}$ using a Molecular Devices, Spectra Max M3 plate reader. All tests were performed in at least triplicate and triplicate controls were included on each plate. The $\%$ cellular viability of each test was calculated using the following formula:

$\%$ cellular viability $=\frac{\text { Abs test sample }-(\text { mean Abs control }- \text { mean Abs blank })}{(\text { mean Abs control }- \text { mean Abs blank })}$

Cellular viability $\leq 50 \%$ of the untreated control indicated toxicity, whereas extracts or controls with $>50 \%$ untreated control viability were deemed to be nontoxic.

\section{Statistical analysis}

Data are expressed as the mean \pm SEM of at least three independent experiments. One way ANOVA was used to calculate differences between the control and treated groups, with a $P$ value $<0.01$ considered to be significant.

\section{RESULTS}

\section{Liquid extraction yields and qualitative phytochemical} screening

Extraction of $1 \mathrm{~g}$ quantities of dried plant material with various solvents yielded dried plant extracts ranging from $79 \mathrm{mg}$ (ethyl acetate extract) to $506 \mathrm{mg}$ (methanolic extract; Table 1). Methanol, water and chloroform each extracted high yields of dried extracted material (506, 484 and $429 \mathrm{mg}$ respectively), whilst ethyl acetate and hexane extracted substantially lower masses (79 and $110 \mathrm{mg}$, respectively). The dried extracts were resuspended in $10 \mathrm{~mL}$ of deionised water (containing 1\% DMSO), resulting in the extract concentrations shown in Table 1. The qualitative phytochemical screening studies showed that methanol and water extracted the widest range and largest relative abundances of phytochemicals in 
Table 1: The mass of dried extracted material, the concentration after resuspension in deionised water (containing $1 \%$ DMSO) and qualitative phytochemical screenings of the $E$. officinalis fruit extracts.

\begin{tabular}{|c|c|c|c|c|c|c|c|c|c|c|c|c|c|c|c|}
\hline 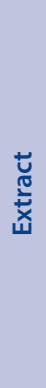 & 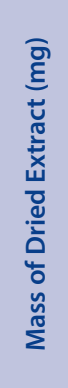 & 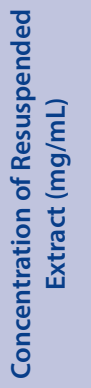 & 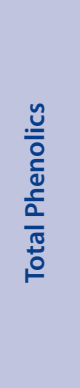 & 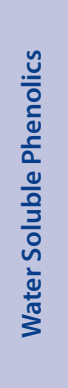 & 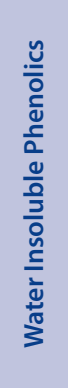 & 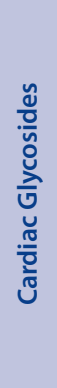 & $\begin{array}{l}\stackrel{n}{\frac{c}{c}} \\
\stackrel{0}{\circ} \\
\stackrel{\circ}{0}\end{array}$ & 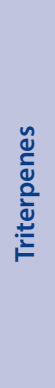 & 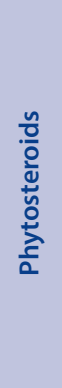 & 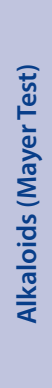 & 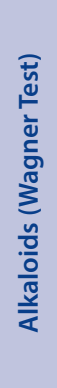 & $\begin{array}{l}\frac{n}{0} \\
\frac{0}{0} \\
\frac{0}{0} \\
\frac{\pi}{4}\end{array}$ & 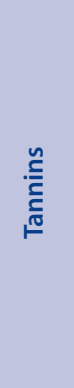 & 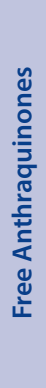 & 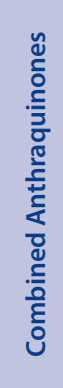 \\
\hline M & 506 & 50.6 & +++ & +++ & +++ & - & +++ & + & + & + & + & +++ & +++ & - & - \\
\hline W & 484 & 48.4 & +++ & +++ & +++ & - & +++ & - & - & + & + & +++ & +++ & - & - \\
\hline E & 79 & 7.9 & +++ & ++ & + & - & + & - & + & - & - & ++ & + & - & - \\
\hline
\end{tabular}

+++ indicates a large response; ++ indicates a moderate response; + indicates a minor response; - indicates no response in the assay; $\mathrm{M}=$ methanolic $\mathrm{E}$. officinalis fruit extract; $\mathrm{W}=$ aqueous $\mathrm{E}$. officinalis fruit extract; $\mathrm{E}=$ ethyl acetate $\mathrm{E}$. officinalis fruit extract; $\mathrm{C}=$ chloroform $\mathrm{E}$. officinalis fruit extract; $\mathrm{H}=$ hexane $\mathrm{E}$. officinalis fruit extract.

this study. Both extracts contained moderate to high levels of phenolic, saponins, flavonoids and tannins. Similar classes of phytochemicals were detected in the ethyl acetate and chloroform extracts, although at substantially lower levels. The methanolic and aqueous extracts also contained low amounts of triterpenoids, phytosterols and alkaloids.

\section{Antibacterial activity}

To examine the growth inhibitory activity of the E. officinalis fruit extracts, a series of disc diffusion assays were conducted on agar plates inoculated with autoimmune inflammatory disease initiating bacterial strains (P. mirabilis, K. pneumoniae, A. baylyi and P. aeruginosa). P. mirabilis growth was susceptible to the methanolic and ethyl acetate E. officinalis fruit extracts (Figure 1), with zones of inhibition of approximately 15-17 mm. notably, these extracts produced substantially larger zones of inhibition than the penicillin and nystatin controls, and comparable inhibition zones as the chloramphenicol control. Indeed, this P. mirabilis strain was completely resistant to nystatin. In contrast, ciprofloxacin was a particularly potent inhibitor of $P$. mirabilis growth. Lower growth inhibitory activity was observed for the aqueous, chloroform and hexane extracts.

A similar trend was noted for inhibition of the bacterial trigger of ankylosing spondylitis (K. pneumoniae), although all the extracts were substantially less potent inhibitors of this bacterium. The E. officinalis fruit ethyl acetate extract was the strongest $K$. pneumoniae growth inhibitor, with an inhibition zone of $8.6 \pm 0.6 \mathrm{~mm}$ (Figure 2). Whilst the ethyl acetate extract was substantially less potent against $K$. pneumoniae than it was against P. mirabilis, this result is still noteworthy as the K. pneumoniae strain tested in these studies was resistant to penicillin and nystatin, and has previously been reported to be resistant to multiple other antibiotics. ${ }^{24}$ In contrast, this bacterium was highly susceptible to chloramphenicol and ciprofloxacin, with zones of inhibition between 21 and $26 \mathrm{~mm}$. The mid to high polarity E. officinalis methanolic, aqueous and ethyl acetate fruit extracts were also strong inhibitors of $A$. baylyi (Figure 3a) and P. aeruginosa growth (Figure $3 \mathrm{~b}$ ).

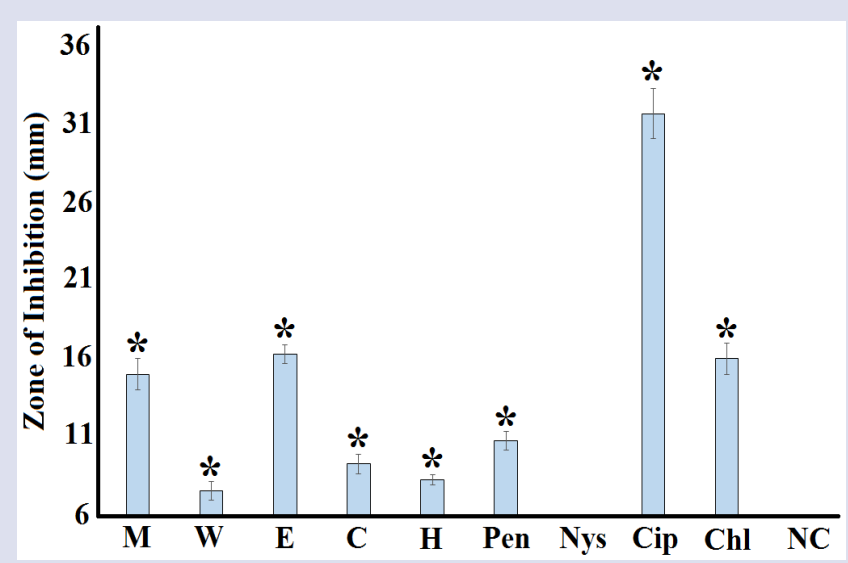

Figure 1: Antibacterial activity of the $E$. officinalis fruit extracts against P. mirabilis (ATCC: 21721) measured as zones of inhibition (mm).

$\mathrm{M}=$ methanolic extract; $\mathrm{W}=$ aqueous extract; $\mathrm{E}=$ ethyl acetate extract; $\mathrm{C}=$ chloroform extract; $\mathrm{H}=$ hexane extract. Positive controls: Pen = penicillin-G $(20 \mu \mathrm{g}) ;$ Nys = nystatin (100 Units); Cip = ciprofloxacin $(2.5 \mu \mathrm{g}) ; \mathrm{Chl}=$ chloramplenicol $(10 \mu \mathrm{g})$. Negative control $(\mathrm{NC})=$ water Results are expressed as mean zones of inhibition of at least six replicates (two repeats) \pm SEM. ${ }^{*}$ indicates results that are significantly different to the negative control $(P<0.01)$.

\section{Quantification of minimum inhibitory concentration (MIC)}

The minimum inhibitory concentration for each E. officinalis extract was determined by further analysing the extracts that showed antimicrobial activity in the disc diffusion screening assays. The extracts were tested across a range of concentrations against $P$. mirabilis, K. pneumonia, A. baylyi and $P$. aeruginosa in microplate liquid dilution and disc diffusion assays to determine the MIC of each extract (Table 2). Antibiotic control MIC's are only provided for the liquid dilution assays as 
the antibiotic standard discs used for the disc diffusion assay were only tested at a single dose. Interestingly, the $P$. aeruginosa strain used in the study was completely resistant to all of the conventional antibiotic controls except chloramphenicol. However, the relatively high MIC value for chloramphenicol against $P$. aeruginosa $(1.25 \mu \mathrm{g} / \mathrm{mL})$ indicates that this bacterium is also resistant to this antibiotic. In addition, both penicillin-G and erythromycin were completely ineffective against $P$. mirabilis, K. pneumoniae and A. baylyi. Whilst chloramphenicol and tetracycline did inhibit P. mirabilis, K. pneumoniae and A. baylyi growth, their relatively high MIC values indicate that all of the bacteria were partially resistant to all of the conventional antibiotics screened in this study.

The ethyl acetate extract generally displayed the lowest MIC against all bacterial strains. The ethyl acetate extract was a potent inhibitor of P. aeruginosa, (DD MIC $264 \mu \mathrm{g} / \mathrm{mL}$; LD MIC $523 \mu \mathrm{g} / \mathrm{mL}$ ). Interestingly, despite its resistance to the conventional antibiotics, $P$. aeruginosa was

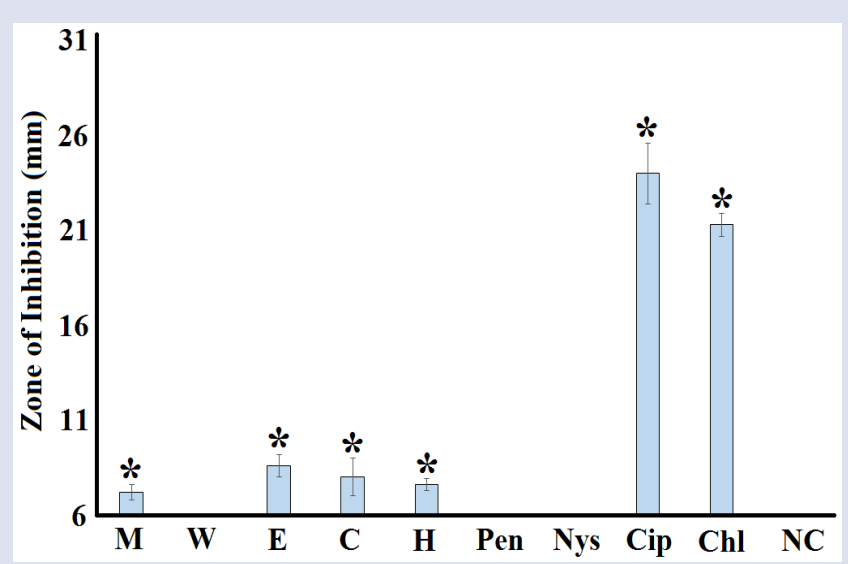

Figure 2: Antibacterial activity of the E. officinalis fruit extracts against K. pneumoniae (ATCC: 39324) measured as zones of inhibition (mm). $\mathrm{M}=$ methanolic extract; $\mathrm{W}=$ aqueous extract; $\mathrm{E}=$ ethyl acetate extract; $\mathrm{C}=$ chloroform extract; $\mathrm{H}=$ hexane extract. Positive controls: Pen =

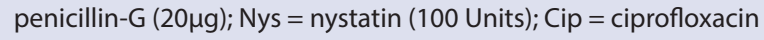
$(2.5 \mu \mathrm{g}) ; \mathrm{Chl}=$ chloramplenicol $(10 \mu \mathrm{g})$. Negative control $(\mathrm{NC})=$ water. Results are expressed as mean zones of inhibition of at least six replicates (two repeats) \pm SEM. * indicates results that are significantly different to the negative control $(\mathrm{P}<0.01)$.
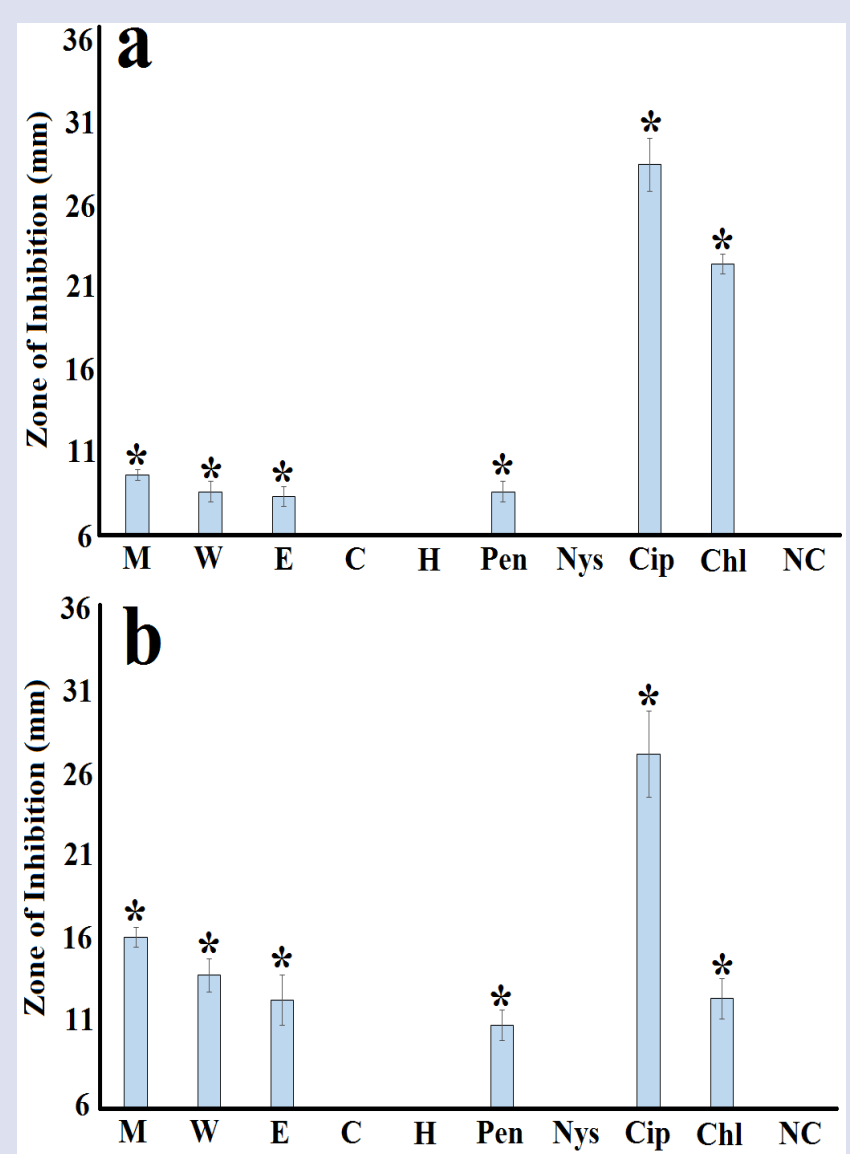

Figure 3: Antibacterial activity of the $E$. officinalis fruit extracts against (a) A. baylyi (ATCC:21721) and (b) P. aeruginosa (ATCC:31488) measured as zones of inhibition $(\mathrm{mm})$. $\mathrm{M}=$ methanolic extract; $\mathrm{W}=$ aqueous extract; $\mathrm{E}=$ ethyl acetate extract; $\mathrm{C}=$ chloroform extract; $\mathrm{H}=$ hexane extract. Positive controls: Pen = penicillin-G $(20 \mu \mathrm{g})$; Nys = nystatin (100 Units); Cip = ciprofloxacin $(2.5 \mu \mathrm{g}) ; \mathrm{Chl}=$ chloramplenicol $(10 \mu \mathrm{g})$. Negative control $(\mathrm{NC})=$ water. Results are expressed as mean zones of inhibition of at least six replicates (two repeats) \pm SEM. * indicates results that are significantly different to the negative control $(P<0.01)$.

Table 2: Disc diffusion and liquid dilution MIC values $(\mu \mathrm{g} / \mathrm{mL})$ against the bacterial triggers of some selected autoimmune inflammatory diseases.

\begin{tabular}{|c|c|c|c|c|c|c|c|c|}
\hline \multirow[t]{3}{*}{ Extract } & \multicolumn{8}{|c|}{ Minimum Inhibitory Concentration $(\mu \mathrm{g} / \mathrm{mL})$} \\
\hline & \multicolumn{2}{|c|}{ P. mirabilis } & \multicolumn{2}{|c|}{ K. pneumoniae } & \multicolumn{2}{|c|}{ A. baylyi } & \multicolumn{2}{|c|}{$P$. aeruginosa } \\
\hline & DD & LD & DD & LD & DD & LD & DD & LD \\
\hline Methanol & 5940 & 5737 & $>10,000$ & 2630 & $>10,000$ & 5737 & 2588 & 2565 \\
\hline Water & 6136 & 5260 & - & 2630 & $>10,000$ & 5260 & 3125 & 2630 \\
\hline Ethyl acetate & 1355 & 1047 & 950 & 1526 & 2258 & 1460 & 264 & 523 \\
\hline Chloroform & $>10,000$ & $>10,000$ & 2615 & 2850 & - & - & - & - \\
\hline Hexane & $>10,000$ & $>10,000$ & 1444 & 860 & - & - & - & - \\
\hline \multicolumn{9}{|l|}{ Positive control } \\
\hline Penicillin & ND & - & ND & - & ND & - & ND & - \\
\hline Erythromycin & ND & - & ND & - & ND & - & ND & - \\
\hline Chloramphenicol & ND & 1.25 & ND & 0.63 & ND & 1.25 & ND & 1.25 \\
\hline Tetracycline & ND & 0.63 & ND & 0.63 & ND & 0.63 & ND & - \\
\hline
\end{tabular}

The values represent the MIC value in $\mu \mathrm{g} / \mathrm{mL}$. $\mathrm{DD}=$ disc diffusion; $\mathrm{LD}=$ liquid dilution; $\mathrm{ND}=$ not determined; - = no inhibition was observed. 
Hutchings, et al.: Amla inhibits bacterial triggers of some autoimmune diseases

Table 3: $\Sigma F I C$ values for combinations of the $E$. officinalis fruit extracts in combination with chloramphenicol and tetracycline against the bacterial triggers of some autoimmune inflammatory diseases.

\begin{tabular}{|c|c|c|c|c|c|}
\hline \multirow[t]{2}{*}{ Antibiotic } & \multirow[t]{2}{*}{ Extract } & \multicolumn{4}{|c|}{ 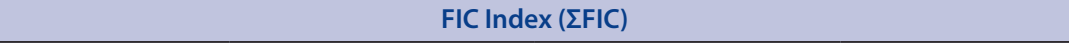 } \\
\hline & & P. mirabilis & K. pneumoniae & A. baylyi & P. aeruginosa \\
\hline \multirow[t]{5}{*}{ Chloramphenicol } & Methanol & 0.63 & 0.75 & 0.75 & - \\
\hline & Water & 0.75 & 1.00 & 0.63 & - \\
\hline & Ethyl acetate & 1.00 & 0.75 & 1.12 & - \\
\hline & Chloroform & - & - & - & 0.75 \\
\hline & Hexane & - & - & - & - \\
\hline \multirow[t]{5}{*}{ Tetracycline } & Methanol & 0.83 & 1.0 & 0.75 & - \\
\hline & Water & 0.95 & 1.0 & 0.63 & - \\
\hline & Ethyl acetate & 1.25 & 0.75 & 1.25 & - \\
\hline & Chloroform & - & - & - & - \\
\hline & Hexane & - & - & - & - \\
\hline
\end{tabular}

Numbers indicate the mean $\Sigma$ FIC values of 6 determinations; - (grey highlighting) = could not be determined as one or both components of the combination were inactive; Additive (blue highlighting) $=\Sigma$ FIC $>0.5-\leq 1.0$; Indifferent effects (green highlighting) $=>1.0-\leq 4.0$.

the most susceptible bacteria to all of the high to mid polarity extracts, with MIC values generally in the $1000-1500 \mu \mathrm{g} / \mathrm{mL}$ range against all other bacterial species. This is particularly noteworthy as this $P$. aeruginosa strain was resistant to all conventional antibiotics tested in this assay, indicating that the bioactive components within these extracts may function via different mechanisms to these antibiotics. Alternatively, the extracts may contain compounds which potentiate the antibacterial components, allowing them to function in bacteria that would be otherwise resistant to their actions. The methanolic and aqueous extracts also inhibited bacterial growth. However, the measured MIC values indicate only moderate to weak inhibition of $P$. mirabilis, $K$. pneumoniae and A. baylyi.

\section{Determination of combinational effects: Fractional inhibitory concentration (FIC) assessment}

Fractional inhibitory concentration (FIC) determination was performed using a 1:1 ratio of each E. officinalis extract and conventional antibiotic and sums of FIC ( FIC) were calculated (Table 3 ). As all bacterial species were completely resistant to penicillin-G and erythromycin in the liquid dilution MIC assay, no $\Sigma$ FIC values could be calculated for combinations containing these antibiotics. Only the $\Sigma$ FIC values for combinations containing chloramphenicol and tetracycline could be calculated. Furthermore, as P. aeruginosa (ATCC:31488) was only susceptible to chloramphenicol, $\Sigma$ FIC values could not be determined for the tetracycline containing combinations against $P$. aeruginosa. $\Sigma$ FIC values were calculated for the 19 remaining chloramphenicol or tetracycline containing combinations and the class of interaction was determined. No synergistic interactions were noted for any combination against any bacterial species. Sixteen of the 19 combinations produced additive interactions. These are promising results as the antibacterial activity of these combinations was substantially elevated, albeit not to the extent than expected for a synergistic interaction. However, the increased growth inhibitory activity of these combinations indicates that they would be preferable as growth inhibitors of these bacteria compared to treatment with the antibiotic or extract components alone. Of the remaining combinations, 3 were determined to be non-interactive. Whilst co-administering these extracts and antibiotics would be of no therapeutic benefit, they would not counteract/lessen each other's activity and therefore are safe to use together. Although $\Sigma$ FIC values could not be determined for the remaining interactive combinations (Table 3 ), it is noteworthy that no antagonistic interactions were detected, indicating that the E. officinalis extracts can be used in combination with the conventional antibiotics tested in this study without reducing the effects of the antibiotic component of the combinations. Therefore, none the E. officinalis: antibiotic combinations need to be avoided to maintain antibacterial activity.

\section{Quantification of toxicity}

To evaluate and quantify the effect of the extracts on the induction of mortality, each was diluted in artificial seawater to test across a range of concentrations in the Artemia nauplii bioassay. Table 4 shows the LC $_{50}$ values of the extracts towards A. franciscana. No $\mathrm{LC}_{50}$ values are reported for the ethyl acetate and hexane extracts as $<50 \%$ mortality was seen for all concentrations tested. The methanolic, aqueous and chloroform extracts were also determined to be nontoxic, with $\mathrm{LC}_{50}$ values substantially greater than $1000 \mu \mathrm{g} / \mathrm{mL}$ following $24 \mathrm{~h}$ exposure. Extracts with an $\mathrm{LC}_{50}$ of greater than $1000 \mu \mathrm{g} / \mathrm{mL}$ towards Artemia nauplii have been defined as being nontoxic. ${ }^{31}$ Similarly, the HDF cell viability was $>50 \%$ for all extract treatments, confirming that all were nontoxic.

\section{DISCUSSION}

This study investigated the ability of E. officinalis fruit extracts to inhibit the growth of some bacterial triggers of autoimmune inflammatory diseases, both alone and in combination with conventional antibiotics. Whilst several extracts were identified as effective growth inhibitors on their own against some bacteria, the majority were found to have only

Table 4: Toxicity determination of the E. officinalis fruit extracts in the Artemia nauplii (ALA) and human dermal fibroblast (HDR) assay following $24 \mathrm{~h}$ exposure.

\begin{tabular}{ccc}
\hline \multirow{2}{*}{ Extract } & \multicolumn{2}{c}{ Toxicity } \\
\cline { 2 - 3 } & ALA $(\mu \mathrm{g} / \mathrm{mL})$ & HDF assay \\
\hline Methanol & 1655 & NT \\
Water & 2830 & NT \\
Ethyl acetate & - & NT \\
Chloroform & 4420 & NT \\
Hexane & - & NT \\
\hline
\end{tabular}

- indicates that $<50 \%$ mortality was induced at all concentrations tested. NT $=$ not toxic ( $>50 \%$ cell viability) in the HDR assay 
low to moderate activity. Thus, most of the E. officinalis fruit extracts tested in our study generally do not have clinically relevant potency and have limited therapeutic value. This is in contrast to the findings of a previous study which described aqueous E. officinalis fruit extracts as being potent inhibitors of bacterial growth. ${ }^{15}$ However, the definition of potency as applied to the evaluation of the strength of the extracts by the authors' of that study was based entirely on comparisons between the zones of inhibition produced by the relatively higher concentrations of the E. officinalis fruit extracts screened. The authors did report substantial inhibition zones of up to $20 \mathrm{~mm}$ (including the $8 \mathrm{~mm}$ diameter well). However, the extracts were tested undiluted and at a single dose. Furthermore, the concentrations of the extracts were not determined, making any evaluation of the strength of inhibition impossible. Additionally, the Saeed and Tariq study ${ }^{15}$ lacked the inclusion of any positive controls, raising further questions about the relevance of their results. It is noteworthy that the antibacterial screens presented in our study (which also used undiluted extracts) had similar sized zones of inhibition against some bacterial species using a $10 \mu \mathrm{L}$ disc load (compared to the $100 \mu \mathrm{L}$ volumes loaded into the wells in the Saeed and Tariq study). ${ }^{15}$ Despite the large inhibition zones measured for the undiluted extracts in our study, when the antibacterial potency was quantified by MIC determination, the values indicated only moderate efficacy against most bacteria. The ethyl acetate extract was the exception, with strong inhibition noted against some pathogens. That extract was a particularly potent inhibitor of $P$. aeruginosa growth, with an MIC of $264 \mu \mathrm{g} / \mathrm{mL}$. This MIC is clinically relevant and indicates that this extract warrants further investigation. As $P$. aeruginosa is a bacterial trigger of multiple sclerosis, the ethyl acetate E. officinalis fruit extract may be useful in the prevention and treatment of that disease (as well as other diseases caused by that bacterium). Weaker, albeit still clinically relevant potency was also noted against $P$. mirabilis, K. pneumoniae and A. baylyi, indicating that the ethyl acetate E. officinalis fruit extract may also be useful in treating and preventing rheumatoid arthritis and ankylosing spondylitis in genetically susceptible people.

Aside from the different techniques used between our report and the aforementioned study, as well as the differences in interpretation, a number of other factors may have also contributed to the apparently different efficacies. The Saeed and Tariq study. ${ }^{15}$ screened against different bacterial pathogens to those tested in our study. Different bacterial species may have very different susceptibilities, which could explain the apparent differences in potency. Furthermore, the previous study screened against clinical bacterial isolates. Bacterial susceptibility to antibiotics and to plant extracts may vary widely between different strains of bacteria, even within the same species. ${ }^{1}$ Mutations may occur in bacterial DNA, providing the cell resistance to specific antibiotic(s). Alternatively, bacterial cells may incorporate DNA from other bacteria in their environment via conjugation or transformation and also develop resistance. When clinical or environmental bacterial isolates are used, little is known about their susceptibilities and/or resistances so wide discrepancies are possible between different studies. For this reason, our study has focussed on well characterised reference bacterial strains to allow for comparison to other studies. Variation may also occur between the plant materials examined in the different studies. Factors such as the geographical location of the plant's collection, the age of the plant, nutrient concentrations due to soil and environmental growth conditions, and ultimately the extraction method, can affect the final yield and levels of phytoconstituents present in a plant, and thus have substantial effects on the levels of activity measured. It is noteworthy that the Saeed and Tariq study. ${ }^{15}$ only screened against aqueous extracts (both decoctions and infusions), whereas our study tested several extracts of varying polarity. Interestingly, our study determined the aqueous extract to have relatively low potency compared to the other extracts.
We also investigated the effects of combinations of the E. officinalis fruit extracts with conventional antibiotics on antibacterial activity. Whilst none of the combinations were synergistic, sixteen combinations produced additive effects. Indeed, additive results was obtained against all 4 bacterial strains tested in this study. These combinations would be beneficial for individuals suffering these autoimmune diseases as they would provide enhanced antibacterial efficacy compared to treatment with either the antibiotic or extract components alone. The use of combinations of $E$. officinalis fruit extracts with conventional antibiotics may also delay the onset of further antibiotic drug resistance, enabling the antibiotic to be used for a longer period of time before it loses clinical relevance. Furthermore, no antagonistic interactions were detected. A further three combinations were determined to be non-interactive and no antagonistic combinations were noted. Thus, whilst not having beneficial effects above those of the individual components, co-administration of the extracts with the conventional antibiotics in these combinations will not lessen the efficacy of the conventional therapies. This information is important as many individuals self-medicate with herbal and traditional medicines and it is therefore important to understand how medicines interact.

Whilst an examination of the phytochemistry of the E. officinalis fruit extracts was beyond the scope of our study, high relative abundances of tannins and flavonoids were noted in the qualitative phytochemical screening studies. This is consistent with previous studies which have reported that the fruits of E. officinalis contain high levels of the tannins gallic acid, 3-ethylgallic acid, ellagic acid, chebulinic acid, chebulagic acid and corilagin. ${ }^{14}$ Gallotannins have been reported to inhibit the growth of a broad spectrum of bacterial species ${ }^{32}$ through a variety of mechanisms including binding cell surface molecules including lipotoichoic acid and proline-rich cell surface proteins, ${ }^{33,34}$ and by inhibiting glucosyltransferase enzymes. ${ }^{35}$ Elligitannins are also highly potent inhibitors of bacterial growth, with MIC values as low as $62.5 \mu \mathrm{g} / \mathrm{ml} .{ }^{32}$ Ellagitannins have also been reported to function via several antibiotic mechanisms including interaction with cytoplasmic oxidoreductases and by disrupting bacterial cell walls. ${ }^{32,34}$ Similarly, E. officinalis fruit also contains appreciable levels of the flavonoids quercetin, kaempferol and apigenin. ${ }^{13}$ Many studies have also reported potent antibacterial activities for a wide variety of flavonoids against a broad panel of pathogenic bacteria. ${ }^{36}$ Indeed, that report highlighted the growth inhibition of a methicillin resistant Staphylococcus aureus (MRSA) by flavonoids, indicating that this extract may have wider therapeutic applications than those reported in the present study. It is therefore likely that the E. officinalis extract flavonoids may contribute to the potent bacterial growth inhibitory activity reported in our study.

It is possible that other phytochemical classes may also contribute to the growth inhibitory properties of these extracts. Our qualitative phytochemical screening studies indicate that polyphenols, saponins, triterpenes, alkaloids and saponins were present in the E. officinalis fruit. Terpenoids have been previously reported to have potent broad spectrum antibacterial activity ${ }^{36}$ and therefore may contribute to the growth inhibitory activity against the bacterial triggers of autoimmune inflammatory diseases tested in our study. Further phytochemical evaluation studies and bioactivity driven isolation of active components is required to evaluate the mechanism of bacterial growth inhibition. The findings reported here also demonstrate that all of the E. officinalis fruit extracts were nontoxic However, whilst our preliminary toxicity studies indicate that these extracts may be safe for use as bacterial growth inhibitors, further studies using an extended panel of human cell lines is warranted to evaluate the safety of these extracts. 
Hutchings, et al.: Amla inhibits bacterial triggers of some autoimmune diseases

\section{CONCLUSION}

The ethyl acetate E. officinalis extract (and the methanolic extract to a lesser extent) was a good inhibitor of the growth of the autoimmune bacterial triggers. It was especially potent against $P$. aeruginosa, indicating that it would be particularly useful for the prevention and treatment of multiple sclerosis. Additive interactions between these extracts in combination with either chloramphenicol or tetracycline indicate that these combinations may provide enhanced therapeutic outcomes and more studies are warranted.

\section{ACKNOWLEDGEMENT}

Financial support for this work was provided by the Environmental Futures Research Institute and the School of Natural Sciences, Griffith University, Australia.

\section{CONFLICT OF INTEREST}

The authors report no conflicts of interest.

\section{ABBREVIATIONS}

DMSO: Dimethyl sulfoxide; $\mathbf{L C}_{50}$ : The concentration required to achieve $50 \%$ mortality; MIC: minimum inhibitory concentration; FIC: Fractional inhibitory concentration; EFIC: Sum of fractional inhibitory concentrations.

\section{REFERENCES}

1. Cheesman MJ, llanko A, Blonk B, Cock IE. Developing new antimicrobial therapies: Are synergistic combinations of plant extracts/compounds with conventional antibiotics the solution? Pharmacognosy Reviews. 2017;11(22):57-72. DOI: 10.4103/phrev.phrev_21_17.

2. WHO. Antimicrobial resistance. World Health Organization. Available from: http://www.who.int/mediacentre/factsheets/fs194/en/. [Last cited on 2017 May 10]. 2016.

3. Davies J. Where have all the antibiotics gone? Canadian Journal of Infectious Diseases and Medical Microbiology. 2006;17(5):287-90.

4. WHO. The evolving threat of antimicrobial resistance: Options for action. World Health Organization 2014.http://apps.who.int/iris/bitstream/10665/44812/ 1/9789241503181_eng.pdf. Accessed 14 March 2017.

5. Van Vuuren S, Viljoen A. Plant-based antimicrobial studies-methods and approaches to study the interaction between natural products. Planta Medica. $2011 ; 77(11): 1168-82$.

6. Abreu AC, McBain AJ, Simoes M. Plants as sources of new antimicrobials and resistance-modifying agents. Natural Product Reports. 2012;29(9):1007-21.

7. Cottarel G, Wierzbowski J. Combination drugs, an emerging option for antibacterial therapy. Trends in Biotechnology. 2007;25(12):547-55.

8. Chowdhury AN, Ashrafuzzaman M, Ali H, Liza LN, Zinnah KMA. Antimicrobial activity of some medicinal plants against multi drug resistant human pathogens. Advances in Bioscience and Bioengineering. 2013;1(1):1-24.

9. Cock IE. The medicinal properties and phytochemistry of plants of the genus Terminalia (Combretaceae). Inflammopharmacology. 2015;23(5):203-29. DOI: 10.1007/s10787-015-0246-z.

10. Belapurkar P, Goyal P, Tiwari-Barua P. Immunomodulatory effects of Triphala and its individual constituents: A review. Indian Journal of Pharmaceutical Sciences. 2014;76(6):467-75.

11. Chainani S, Siddana S, Reddy C, Thippeswamy M, Maurya M, Rudraswamy S. Antimicrobial activity of Triphala on Lactobacilli and Candida albicans: An in vitro study. Journal of Orofacial Sciences. 2015;7(2):104-7.

12. Khan KH. Roles of Emblica officinalis in medicine - A review. Botany Research International. 2009;2(4):218-28.

13. Habib RKA, Yasin MA, Choudhary N, Khaliq A, Choudhary MI, Malik S. Studies on the chemical constituents of Phyllanthus emblica. Natural Product Research. 2007;21(9):775-81.

14. Zhang LZ, Zhao WH, Guo YJ, Tu GZ, Lin S, Xin LG. Studies on chemical constituents in fruits of Tibetan medicine Phyllanthus emblica. China Journal of Chinese Materia Medica. 2003;28(10):940-3.
15. Saeed S, Tariq P. Antimicrobial activities of Embilica officinalis and Coriandrum sativum against gram positive bacteria and Candida albicans. Pakistan Journal of Botany. 2007;39(3):913-7.

16. Courtney R, Sirdaarta J, Matthews B, Cock IE. Tannin components and inhibitory activity of Kakadu plum leaf extracts against microbial triggers of autoimmune inflammatory diseases. Pharmacognosy Journal. 2015;7(1):18-31. DOI: 10.5530/ pj.2015.7.2.

17. Cock IE, Cheesman M. The potential of plants of the genus Syzygium (Myrtaceae) for the prevention and treatment of arthritic and autoimmune diseases. In Bioactive Foods as Dietary Interventions for Arthritis, Osteoarthritis, and Related Autoimmune Siseases. $2^{\text {nd }}$ edition. 2018. Edited by Preedy VR, Watson RR: Elsevier, Netherlands.

18. Boyer $\mathrm{H}$, Cock IE. Evaluation of the potential of Macademia integriflora extracts as antibacterial food agents. Pharmacognosy Communications. 2013;3(3):53-62. DOI: $10.5530 / p c .2013 .3 .10$.

19. Vesoul J, Cock IE. The potential of Bunya nut as an antibacterial food agent. Pharmacognosy Communications. 2012;2(1):72-9.

20. Winnett V, Boyer H, Sirdaarta, J, Cock IE. The potential of Tasmannia lanceolata as a natural preservative and medicinal agent: Antimicrobial activity and toxicity. Pharmacognosy Communications. 2014;4(1):42-52. DOI: 10.5530/pc.2014.1.7.

21. Cock IE, Van Vuuren S. Anti-Proteus activity of some South African medicinal plants: Their potential for the treatment and prevention of rheumatoid arthritis. Inflammopharmacology. 2014;22(1):23-36. DOI 10.1007/s10787-013-0179-3.

22. Cock IE. Antimicrobial activity of Acacia aulacocarpa and Acacia complanta methanolic extracts. Pharmacognosy Communications. 2012;2(1):66-71.

23. Hubsch Z, Van ZRL, Cock IE, Van Vuuren S. Interactive antimicrobial and tox icity profiles of conventional antimicrobials with Southern African medicinal plants. South African Journal of Botany. 2014;93:185-97. DOI: 10.1016/j. sajb.2014.04.005

24. Ilanko A, Cock IE. The interactive antimicrobial activity of contentional antibiotics and Petalostigma spp. Extracts against bacterial triggers of some autoimmune inflammatory diseases. BMC Complementary and Alternative Medicine. 2018. in press.

25. Arkhipov A, Sirdaarta J, Rayan P, McDonnell PA, Cock IE. An examination of the antibacterial, antifungal, anti-Giardial and anticancer properties of Kigelia africana fruit extracts. Pharmacognosy Communications. 2014;4(3):62-76. DOI: 10.5530/pc.2014.3.7.

26. Hart C, Illanko P, Sirdaarta J, Rayan P, McDonnell PA, Cock IE. Tasmannia stipitata as a functional food/natural preservative: Antimicrobial activity and toxicity. Pharmacognosy Communications. 2014;4(4):33-47. DOI: 10.5530/pc.2014.4.4.

27. Mpala L, Chikowe G, Cock IE. No evidence of antiseptic properties and low toxicity of selected Aloe species. Journal of Pharmaceutical Negative Results. 2010;1(1):10-6. DOI: 10.4103/0976-9234.68869.

28. Sirdaarta J, Matthews B, Cock IE. Kakadu plum fruit extracts inhibit the growth of the bacterial triggers of rheumatoid arthritis: Identification of stilbene and tannin components. Journal of Functional Food. 2015;17:610-20. DOI: 10.1016/j. jff.2015.06.019.

29. Wright $M H$, Sirdaarta J, White A, Greene AC, Cock IE. GC-MS headspace analysis of Terminalia ferdinandiana fruit and leaf extracts which inhibit Bacillus anthracis growth. Pharmacognosy Journal. 2017;9(1):73-82. DOI: 10.5530/ pj.2017.1.14.

30. Shalom J, Cock IE. Terminalia ferdinandiana Exell. fruit and leaf extracts inhibit proliferation and induce apoptosis in selected human cancer cell lines. Nutritrion and Cancer. 2018. in DOI: 10.1080/01635581.2018.1460680

31. Ruebhart DR, Wickramasinghe W, Cock IE. Protective efficacy of the antioxidants vitamin $\mathrm{E}$ and Trolox ${ }^{\mathrm{TM}}$ against Microcystis aeruginosa, microcystin-LR and menadione toxicity in Artemia franciscana nauplii. Journal of Toxicology and Environmental Health Part A. 2009;72(24):1567-75.

32. Buzzini P, Arapitsas P, Goretti M, Branda E, Turchetti B, Pinelli P, et al. Antimicrobial activity of hydrolysable tannins. Mini-Reviews in Medicinal Chemistry. 2008;8(12):1179-87.

33. Wolinsky LE, Sote EO. Isolation of natural plaque-inhibiting substances from 'Nigerian chewing sticks'. Caries Research. 1984;18(3):216-25.

34. Hogg SD, Embery G. Blood-group-reactive glycoprotein from human saliva interacts with lipoteichoic acid on the surface of Streptococcus sanguis cells. Archives in Oral Biology. 1982;27(3):261-8.

35. Wu-Yuan CD, Chen CY, Wu RT. Gallotannins inhibit growth, water-soluble glucan synthesis, and aggregation of Streptococci mutans. Journal of Dental Research. 1988;67(1):51-5.

36. Cock IE. The phytochemistry and chemotherapeutic potential of Tasmania lanceolata (Tasmanian pepper): A review. Pharmacognosy Communications. 2013;3(4):1-13. DOI: 10.5530/pc.2013.4.3 
GRAPHICAL ABSTRACT

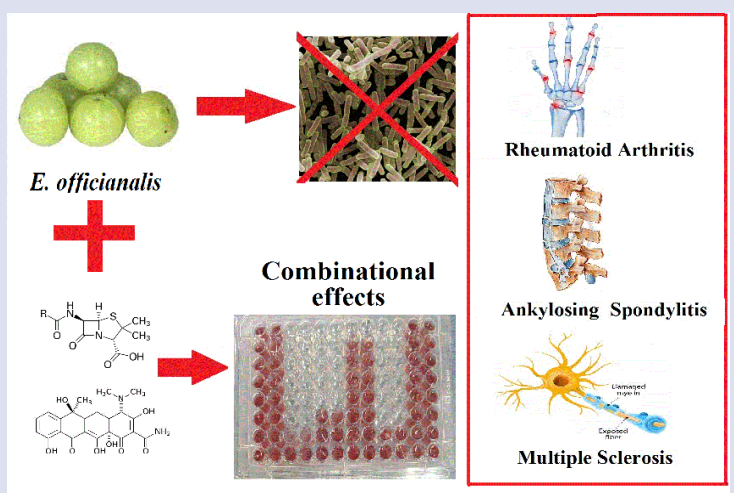

\section{SUMMARY}

- E. officinalis fruit extracts inhibited the growth of some bacterial triggers of autoimmune inflammatory disease

- The ethyl acetate (EA) extract was a particularly potent inhibitor of $P$. aeruginosa growth (MIC $=264 \mu \mathrm{g} / \mathrm{mL})$.

- The EA extract was also a moderate inhibitor of $P$. mirabilis, $K$. pneumonia and A. baylyi, Growth (MIC values 1000-1500 $\mu \mathrm{g} / \mathrm{mL}$ ).

- Methanolic and aqueous extracts also inhibited the growth of all bacteria with moderate to low activity.

- The extracts also potentiated the activity of chloramphenicol and tetracycline against otherwise resistant bacterial strains.

- All extracts were nontoxic in the Artemia nauplii and HDF toxicity assays, further indicating their potential for medicinal use.

\section{ABOUT AUTHORS}

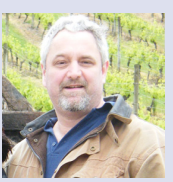

Dr lan Cock: Leads a research team in the Environmental Futures Research Institute and the School of Natural Sciences at Griffith University, Australia. His research involves bioactivity and phytochemical studies into a variety of plant species of both Australian and international origin, including Aloe vera, South Asian and South American tropical fruits, as well as Australia plants including Scaevola spinescens, Pittosporum phylliraeoides, Terminalia ferdinandiana (Kakadu plum), Australian Acacias, Syzygiums, Petalostigmas and Xanthorrhoea johnsonii (grass trees). This range of projects has resulted in nearly 200 publications in a variety of peer reviewed journals. 